\title{
The formation and stability of bulk amorphous and high entropy alloys
}

\author{
Attila SZABÓ, ${ }^{1}$ Krisztián BÁN, ${ }^{2}$ József HLINKA, ${ }^{3}$ Judit PÁSZTOR, ${ }^{4}$ Antal LOVAS ${ }^{5}$ \\ ${ }^{1}$ University of Dunaujvaros, Department of Mechanical Engineering, Dunaujvaros, Hungary, \\ szaboattila@uniduna.hu \\ ${ }^{2}$ Budapest University of Technology and Economics, Faculty of Transportation Engineering, Department \\ of Automotive technologies, Budapest, Hungary, ban.krisztian@kjk.bme.hu \\ ${ }^{3}$ Budapest University of Technology and Economics, Faculty of Transportation Engineering, Department \\ of Automotive technologies, Budapest, Hungary, hlinka.jozsef@kjk.bme.hu \\ ${ }^{4}$ Sapientia Hungarian University of Transylvania Faculty of Technical and Human Sciences, Târgu-Mures,, \\ Romania, pjudit@ms.sapientia.ro \\ ${ }^{5}$ Budapest University of Technology and Economics, Faculty of Transportation Engineering, Department \\ of Automotive technologies, Budapest, Hungary, antal.lovas@gjt.bme.hu
}

\begin{abstract}
Two kinds of phase stabilization mechanism are discussed and compared: the first is characteristic of the formation of bulk amorphous alloys, in which the high supercooling ability of multicomponent liquids is responsible for the glassy phase stabilization. Here the hindered nucleation of crystalline phases is the center phenomenon. The origin of this hindering is the slowing atomic mobility in the supercooling melt. In contrast the melt supercooling is negligible during the high entropy alloy formation. It is believed that stability of the crystalline single fcc phase is the consequence of the characteristic of high configurational entropy at high temperatures. However, the significance of this entropy-dominated stabilization is overestimated in several references. It has been concluded that transition metal contraction (arising from the delectron participation in the overall bonding state) does also contribute to the high temperature stability of fcc single phase in the high entropy alloys.
\end{abstract}

Keywords: bulk metallic glass, high entropy alloy.

\section{Introduction}

During the past few decades of metal physics (1980 -2020) the non-equilibrium metallic systems have become the center of interest. Two important magnetic properties: the excellent high frequency magnetic permeability and, the high saturation induction [1] are successfully unified in the glassy alloys, resulting in the development of new types of amorphous alloy transformers and motors with reduced power loss, and several types of other inductive elements [2,3]. Recently, scientific interest has turned towards two groups of materials: the bulk amorphous alloys (BMGs) and the high entropy alloys (HEAs), as these group of materials promise a breakthrough in the improvement of high temperature mechanical properties [4]. The bulk amorphous alloys with high glass forming ability and high phase stability [4] exhibit some similarity to the high entropy alloys [5]: both groups of alloys consist of several chemical elements. The numerous components play a dominant role in the phase stabilization of these materials.

In this paper we point to the significance of the strength of individual chemical bonds in the retardation of atomic diffusivity towards the fully thermodynamic equilibrium structure.

\section{Results and discussion}

\subsection{Similarity and difference in the forma- tion mechanism of BMGs and HEAs}

Both BMGs and HEAs are formed from a liquid state consisting of several chemical components. 
The main difference between them is the degree of melt supercooling ability. The difference between the supercooling results in fundamentally different solidified structures, i.e., the supercooling is a central phenomenon from the point of view of solidified structure.

Bulk Metallic Glasses (BMG) is a class of multi-component metallic materials, at which crystallization is kinetically suppressed by a number of factors such as atomic diameter difference, high natural supercooling, negative heat of constituent elements mixing, etc. Material systems with high Glass-Forming Ability (GFA) are thermodynamically unstable, and then subsequent heating (,turning on” of diffusion) may cause their crystallization.

On the other hand, High Entropy Alloys (HEA) exist in single-phase solid solution, the crystalline state characterized by high configurational entropy. They just have a strong thermodynamically dictated tendency to avoid formation of ordered solid solutions as well as inter-metallic compounds. The main term which is responsible for their stability is the entropy term, so their heating improves their stability.

The supercooling phenomena is illustrated in the Figure 1 where the entropy change is depicted during the cooling of two liquid types. BMGs and HEAs basically differ from the point of view of their supercooling ability, hence also the temperature range of thermodynamic stability of the resulting solid phases also differ. When the single component liquid is cooled, at $T_{m}$ (equilibrium melting point) the liquid starts to crystallize, and crystallization finishes at the same temperature, so a supercooled state does not appear prior to and during crystallization. In this case the total free enthalpy change is identical to the entropy change. Nevertheless, the liquid-crystalline transformation can also happen at significantly lower temperatures between $T_{m}$ and $T_{K}$ where $T_{K}$ is the Kauzmann-temperature, which is the limit of supercooling. In this temperature range non-equilibrium solidification occurs, so the resulting structure is metastable.

It has also been pointed out that the supercooling ability of high entropy alloys in liquid is negligible.

Compared to the liquids of HEAs, the BMGs are able to deeply super-cool. The entropy of liquid within this range approaches the solid phase, and at $T_{k}$ the entropy of the two phases will be identical (Figure 1).

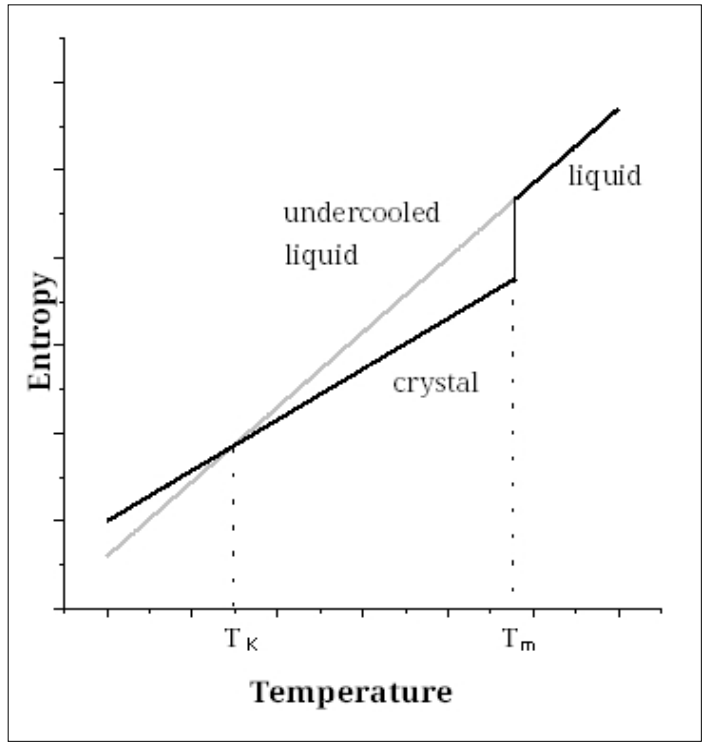

Figure 1. The temperature dependence of entropy in the liquid and crystalline phases: the meaning of Kauzmann temperature. [6]

The degree of supercooling-ability exhibits a great variety in liquids. This phenomenon can be described successfully by the temperature dependence of viscosity. The kinetic aspects in submicroscopic scale is represented by the change of atomic mobility during supercooling: The key concept is the time-scale of elementary atomic motions, which increases as the temperature of liquid decreases, being described by the Stokes-Einstein equation:

$$
\frac{1}{\eta}=\lambda^{2} \cdot\left(\frac{A}{l \cdot k \cdot T}\right)
$$

It expresses phenomenological coupling between displacements in atomic level and viscosity, which can be derived from macroscopic measurements ( $A=$ constant, $k=$ Boltzmann constant, $T$ $=$ absolute temperature, $\lambda$ and $l=$ molecular level distances , $\eta=$ viscosity).

The frequency of elementary step of atomic motion is the vicinity of melting point (around 10-13 $\mathrm{sec}$ ), prolonged residence time (preferential contacts) between hetero-atoms is not developed, so the components distribution is nearly random.

The characteristic of temperature dependence of viscosity $\eta(T)$ is used for the description of dynamic phenomena during the process of super-cooling. Two basic type of T-dependence are known (i.e. two liquid types exist from this point of view (see Figure 2): 


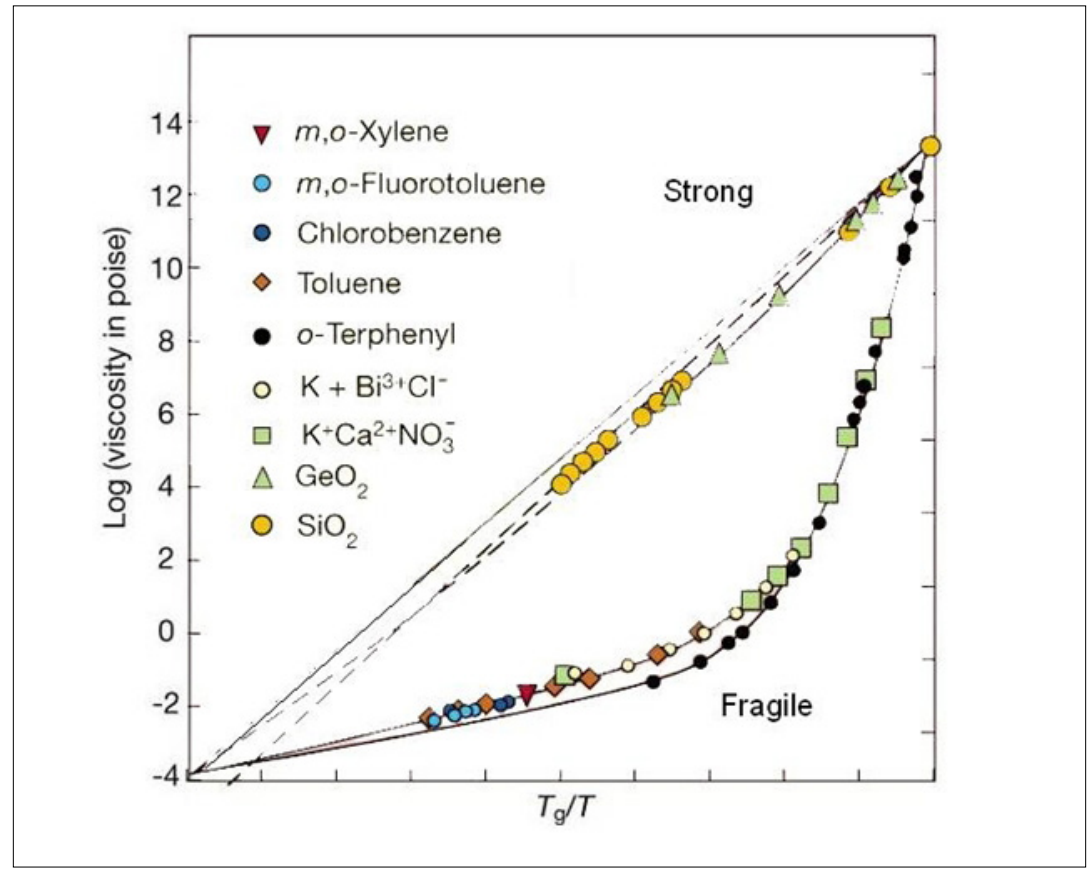

Figure 2. The comparison of $\eta(T)$ for "strong and "fragile" glass forming liquids. [7]

- Arrhenius type:

$\eta=A \cdot \exp \left[E /\left(T-T_{0}\right)\right]$ (strong liquid),

with high GFAm,

- Vogel-Fulcher type:

$\left.\eta=\eta_{0} \cdot \exp \left[B /\left(T-T_{0}\right)\right]\right)$ (fragile liquid)

with low GFAm.

It is believed that Arrhenius functionality with single activation energy represents single motion type in the elementary atomic scale over the whole range of supercooling (strong liquids with high glass forming ability) This high glass forming ability is pronounced in the formation of bulk amorphous alloys. (strong liquids in Figure 2).

In contrast, when two or more motion types coexist and one of them is dominant within a given temperature range, such liquids are termed as „fragile” liquids. The glass forming ability is low in these liquid types. The temperature dependence of viscosity of these liquid types is also illustrated in Figure 2.

From Figures 2 and 3 is clear that the majority of ,strong liquids” are multicomponent alloys, in which the strong covalent bond is dominant between the components (oxide glasses or, multicomponent alloys, with tendency towards intermetallic compound formation in the solid state).

The majority of bulk metallic glasses are formed from Arrhenius-type melts. In Figure 3 different liquids are collected exhibiting the transition between the strong and fragile liquids. All of the liquids are Arrhenius-type at low temperature range. Though, at higher temperatures (beyond $T_{g}$ ) increasing deviation is observed, which strongly correlates with their glass forming ability. In Figure 4 the glass forming ability (the critical cooling rate, $R_{c}$ ) and the resulting maximum of amorphous sample thickness are compared for

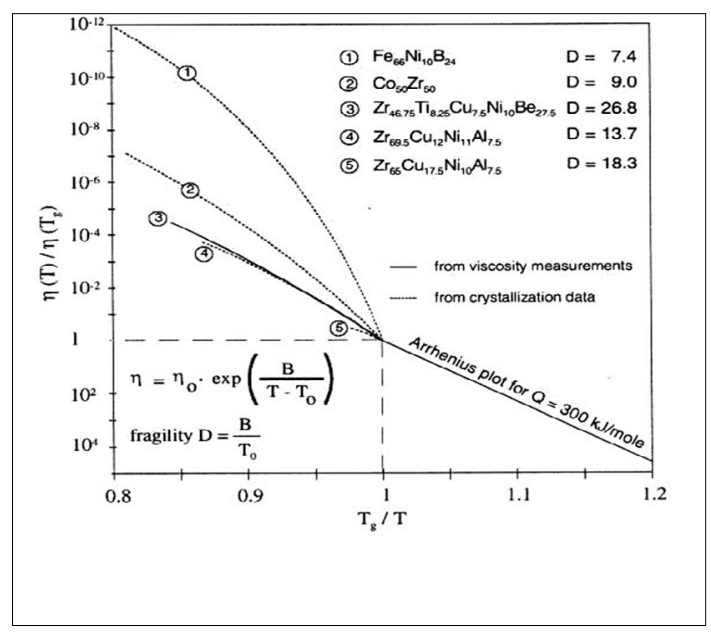

Figure 3. The viscosity temperature dependence for various glassforming ability liquids. [8] 

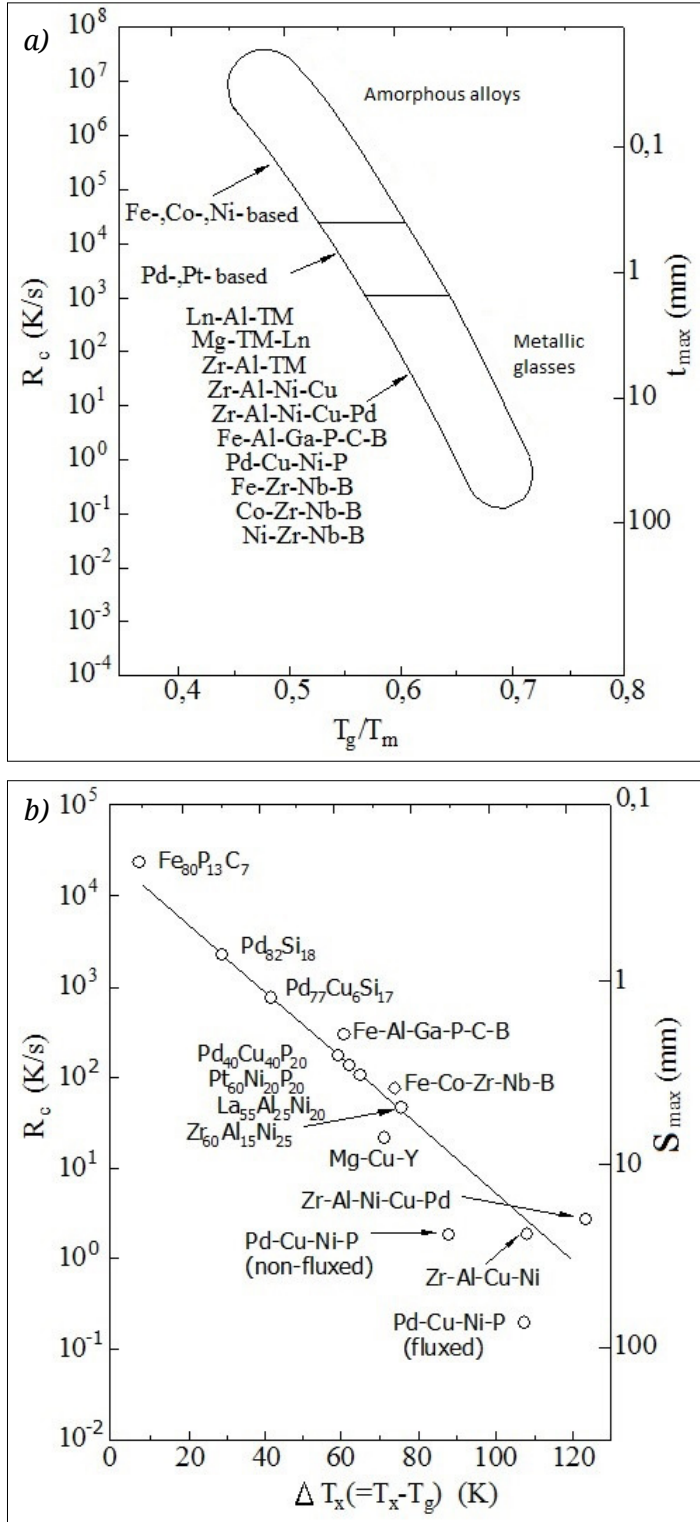

Figure 4. Critical cooling rate and the resulting sample thickness $\left(t_{\max }, S_{\max }\right)$ for several bulk glassy alloys versus the reduced $\left(T_{g} / T_{m}\right)(a)$ or $\Delta T_{X}=T_{x}-T_{g}(b) .[9]$

various bulk amorphous alloys, and soft magnetic amorphous ribbons (produced by rapid solidification).

Compared to the bulk metallic glasses, the glass forming ability for traditional glassy soft magnetic alloys is low, therefore the necessary critical cooling rate is high $(\mathrm{Rc}(\mathrm{K} / \mathrm{s})$ is $\approx 105)$ (see Figure 4).

\subsection{Cluster formation in the glass-forming supercooled liquids}

The interpretation of glass forming ability is in close connection with the cluster formation tendency in liquids [10, 11]. It is supposed that cluster formation in the supercooled melts represents kinetic hindering to the formation of crystalline nuclei. The clusters, which are associated groups of atoms, are considered as a molecule described with $A_{j} B_{j}$ formula, but with a flexible structure in which the atomic distances between the unlike atoms are nearly constant, but the bonding angle is variable over a wide range.

According to the regular solution model conception [12]. such liquid types, the entire mixing enthalpy of alloy formation $(\Delta H$, which can be directly determined, by calorimetry), consists of two terms: $\Delta H^{r e g}$ which is due to the preferential interaction between the hetero-atoms (components), and $\Delta H_{A j B j}^{a s}$ arising from the interaction between the atoms within the associates. This term represents the extra stabilization of the melt, compared to the regular solution model. This consideration leads to the formation of associates, according to (4).

$$
\Delta H=\Delta H^{r e g}+\Delta H_{A j B j}^{a s}
$$

In the first approximation, the structure of supercooled glass-forming melt can be described as a flexible assembly of associates and the remaining components are distributed randomly. The amorphous alloys can be regarded as a cluster assemble, in which the predominantly compound-like bonding state is cumulated [13]. The local bonding state hinders the diffusion leading to the formation of critical crystalline nuclei. The cluster level chemical short range order is developed at sufficient supercooling [14, 15]. As the cooling proceeds, approaching the $\approx 2 / 3 \mathrm{~T}_{\mathrm{m}}$ (jumming temperature, initial temperature of cluster formation) [9], the local composition (TM/M ratio) in compound-like associates is close to that in metastable inter-metallic compound and the local bonding strength between the hetero-atoms is higher. So the translation mobility is lower within the clusters, the time-scale of relaxation inside these covalent-like environments and in the remainder volume of liquid is different.

In conclusion, in the bulk amorphous alloys, which exhibit significant kinetic and thermal stability, the extended clusterization is responsible for the stabilization of amorphous state, due to the local diffusion break down of atoms, hin- 
dering the critical nuclei formation of crystalline phases.

\subsection{The formation mechanism and stability of high entropy alloys (HEA)}

The study of HEA began in 2004. The reason for scientific interest is their high mechanical stability in high temperature ranges coupled with excellent corrosion resistivity. HEAs are also formed from liquid, but their formation does not require a special processing technique. Their denomination originates from the numerous components: the alloys consist of at least five "principal elements" which should have a concentration between 535 at\%. Besides the principal elements HEA can also contain "minor elements" each below 5 at\%. It is believed that high stability originates from the high mixing entropy at high temperatures:

$$
S_{\text {mix }}=R \sum x_{i} \ln x_{i}
$$

where $x_{i}$ is the mole fraction of its component.

In contrast to the bulk amorphous alloys, the HEA are stabilized by configuration entropy at high temperatures. According to $\mathrm{G}=\mathrm{H}-\mathrm{TS}$, (where $\mathrm{G}$ is the Gibbs free energy, $\mathrm{H}$ is enthalpy, $\mathrm{T}$ is temperature, $S$ is entropy) the competition between enthalpy and entropy $S$ determines the phase relation. Hence S can be a stabilizing factor, provided the temperature is sufficiently high. For example, in Co-Cr-Fe-Mn-Ni alloys (which is the best known composition) Ni, Fe, and Co exert a stabilizing effect to the fcc structure, while the opposite structure (bcc) is exerted by $\mathrm{Cr}$ and $\mathrm{Mn}$.

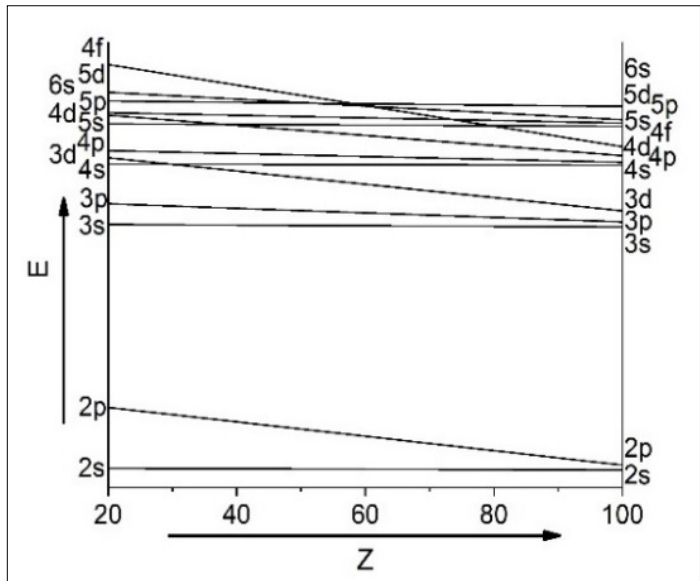

Figure 5. AThe energy level (ionization energy) of $s, p$ and $d$ electrons versus the position in the periodic table. [18]
The mechanism of the resulting preferred high temperature phase stabilization governed by the high entropy is not fully verified yet [16].

Therefore, further activity, directed at a deeper understanding of phase relation, mainly the conditions of single fcc-phase stabilization, is on-going. Recently, some research work has turned to the application of basic metallurgical principles defined earlier by Hume-Rothery [17]. It is surprising, that no correlation is found between the formation enthalpy and the temperature range of the fcc phase stability.

When compared with the ionization energy in the elements of periodic system, (including the s, $\mathrm{p}$, and d electrons) it was found, that ionization energy for the d-electrons is the highest (Figure 5). This means that the bonding strength of d-electrons in the electron band is the highest. Therefore, attractive force between the core and electrons increases, resulting a net contraction of atomic radii.

As a consequence, specially compact, high density structures are developed in the HEAs, when the majority of components are transition metals. In this case, the participation of d-electrons is dominant in the valence band. One can suppose therefore that the well-known principle of transition metal contraction can also participate in the development of dense, random (fcc) structure, when the crystallization from liquid has started. As Figure 6 shows, the atomic radii gradually decrease with the increasing number of $3 d, 4 d$, and $5 \mathrm{~d}$-electrons in the valence band. We can suppose therefore that simultaneous participation of Fe,

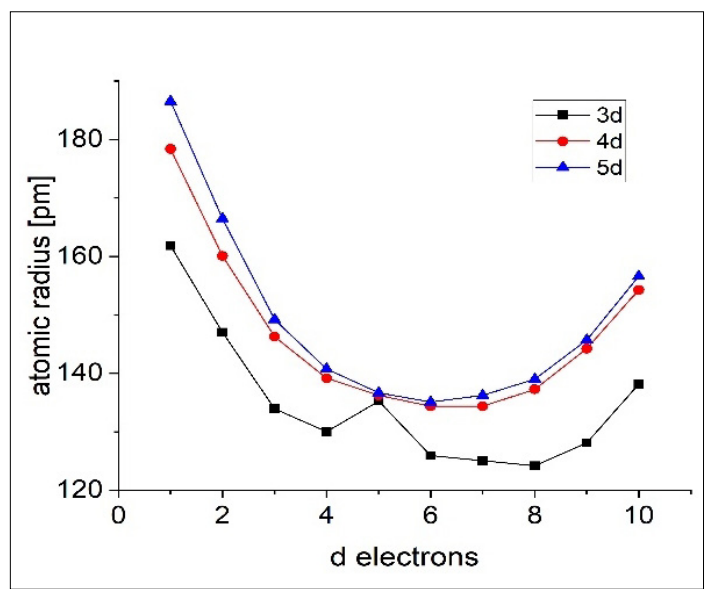

Figure 6. The change of atomic radius versus the increasing number of d-electrons in the valence band in the transition elements. [19] 
Co, Ni principal elements enhance the development of the densest (fcc) structure, therefore, no significant supercooling is required for the critical nuclei formation of fcc solid phases i.e., the stabilization of single phase in wide temperature range.

In spite of the dense structure (which is a disordered solid solution) characteristic of the transition metals, the chemical interaction between the neighbouring TM atoms is not excluded. This interaction does not necessarily result in the formation of inter-metallic compound. This can also manifest as a net local charge shift between various neighbouring atoms, as proposed by Miedema [20], according to (6):

$$
\Delta Z_{a}=1.2 \cdot\left(1-c_{a}\right) \cdot \Delta \Phi^{*}
$$

$\Delta Z_{a}$ charge shift happens between the neighbouring atoms which is determined by the electro-negativity difference between the neighbouring atoms, $\Delta \Phi$ is the difference (work function) ionization potential, which express the electro-negativity difference between two metal components in solid solution, $c_{a}$ is the concentration of the atom $a$.

\section{Conclusions}

Two alloy systems, the BMGs and the single phase HEAs are compared from the point of view of their formation mechanism and their phase stability.

Both alloy systems are formed from liquids, containing more than five components.

Though both alloy systems are multicomponent, the spectra of components exhibit significant differences in their chemical properties: while the BMGs consist of various type of metal as well as metalloids, the main components of HEAs are predominantly transition metals.

While the BMGs are formed from deeply under-cooled liquids, the HEAs solidify in single phase crystalline solid solutions without melt supercooling. This single fcc phase disorder solid solutions fulfilled the high entropy requirement.

While the glassy state in BMGs is stabilized by the extended cluster formation, induced by the strong bonding between the metallic and metalloid components, the stability of HEA originates rather from the strong bonding character of d-electrons due to the dominant participation of d-electrons in the band structure, and resulting dense atomic distribution.

The stability in both systems arises from the local bonding stability between the components (localized covalent-like bonding within the individual clusters). In the case of HEA the strong d-electron-bonding between the atoms of principal elements, results in a dense packing structure.

\section{Acknowledgements}

The research presented in this paper was carried out as part of the EFOP-3.6.2-16-2017-00016 project in the framework of the New Széchenyi Plan. The completion of this project is funded by the European Union and co-financed by the European Social Fund.

The research presented in this paper was carried out as part of the EFOP-3.6.1-16-2016-0003 project in the framework of the New Széchenyi Plan. The completion of this project is funded by the European Union and co-financed by the European Social Fund.

\section{References}

[1] Makino A., Inoue A., Masumoto T.: Nanocrystalline soft magnetic Fe-M-B ( $M=Z r, H f, N b)$ alloys produced by crystallization of amorphous phase (overview). Materials Transactions, JIM, 36/7. (1995) 924-938.

https://doi.org/10.2320/matertrans1989.36.924

[2] Nagy A.: Electric aircraft-present and future. Production Engineering Archives, 23. (2019) 36-40.

[3] Luborsky F. E.: Application oriented magnetic properties. In: Amorphous Metallic Alloys (ed.: Luborsky F. E.) Butterworths Monographs in Materials, London, 1984. 360-379.

[4] Tsai M. H., Yeh J. W.: High-entropy Alloys: A Critical Review. Materials Research Letters, 2/3. (2014) 107-123.

https://doi.org/10.1080/21663831.2014.912690

[5] Inoue A.: Stabilization of Metallic Supercooled Liquid and Bulk Amorphous Alloys. Acta Materialia, 48/1. (2000) 279-306.

https://doi.org/10.1016/S1359-6454(99)00300-6

[6] Kauzman W.: The Nature of the Glassy State and the Behavior of Liquids at Low Temperatures. Chemical Reviews, 43/2. (1948) 219-256.

https://doi.org/10.1021/cr60135a002

[7] Angell C. A.: Structural instability and relaxation in liquid and glassy phases near the fragile liquid limit. Journal of Non-Crystalline Solids, 102/1-3. (1988) 205-221.

https://doi.org/10.1016/0022-3093(88)90133-0

[8] Köster U., Janlewing R.: Fragility parameter and nanocrystallization of metallic glasses. Materials Science and Engineering: A, 375-377 (2004) 223-226.

https://doi.org/10.1016/j.msea.2003.10.083

[9] Inoue A.: Bulk Amorphous Alloys. In: Non-Equilibrium Processing of Materials (ed.: Suryanarayana

C.) Pergamon Materials Series, Pergamon Amsterdam-Lausanne-New York, 1999. 375-415.

[10] Dimitrov V. I.: Theory of Fluidity of Liquids, Glass 
Transition and Melting. Journal of Non-Crystalline Solids, 352/3. (2006) 216-231. https://doi.org/10.1016/j.jnoncrysol.2005.11.026

[11] Lovas A., Ramasamy P. Kováč J., Novák L., Szabó A., Eckert J.: Cluster-related phenomena in the properties and transformations of transition metal-based glassy alloys. Metals, 10/8. (2020) 1025. https://doi.org/10.3390/met10081025

[12] Richardson F. D.: Physical Chemistry of Melts in Metallurgy. Academic Press London, New York, 1. (1974) 142.

[13] Predel B.: Thermodynamic stability of amorphous alloys. Key Engineering Materials, 40-41. (1990) 17-38.

[14] Debenedetti P. G.: Metastable Liquids Concepts and Principles. Princeton Univ. Press, Princeton, 1996.

[15] Debenedetti P. G., Stillinger F. H.: Supercooled liquids and the glass transition. Nature, 410/8. (2001) 259-267.

https://doi.org/10.1038/35065704
[16] Zhang F., Zhang C., Chen S. L., Zhu J., Cao W. S., Kattner U.R.: An understanding of high entropy alloys from phase diagram calculations. CALPHAD, 45. (2014) 1-10.

https://doi.org/10.1016/j.calphad.2013.10.006

[17] Troparevsky M. C., Morris J. R., Daene M., Wang Y., Lupini A. R., Malcolm G.: Beyond Atomic Sizes and Hume-Rothery Rules: Understanding and Predicting High-Entropy Alloys. JOM, 67. (2015) 2350-2363.

https://doi.org/10.1007/s11837-015-1594-2

[18] Barnard A. K., Sykes P.: Theoretical Basis of Inorganic Chemistry. New York (N.Y.): McGraw-Hill, 1965.

[19] Khandelwal B. L.: Inorganic Chemistry of Transition Elements. Disha Institute of Management and Technology, Raipur, 2007.

[20] Miedema A. R., Boom R., De Boer F. R.: On the heat of formation of solid alloys. J. Less-Common Met. 41 (1975) 275-284. 